\title{
Orthopedic Surgeon and Scientific Publications: Understanding the Symbiosis
}

\author{
Dr Imran Sajid, Dr Sandeep Kumar, Dr Javed Jameel, Dr Sukhvinder Singh, \\ Dr Ashok Kumar
}

\begin{abstract}
Scientific publications have always been an integral part of an orthopedic surgeon's curriculum. Publication of one's work in a scientific journal gives an extra edge to a person's curriculum vitae. This article provides basic understanding as to the needs and demands in today's time for scientific presentations. It also provides guidelines about writing a scientific paper and helps in understanding the intricacies related to impact factor, authorship, plagiarism.
\end{abstract}

Keywords: Scientific publication, impact factor, authorship.

\section{Introduction:}

Writing a paper is an art which most of us in scientific community have learned over years by one's own trials and errors. Barring a few fortunate exceptions, writers are made, not born. Presented here are few basic points gathered over the years (by the authors), who believe that a well written; published scientific paper is the medium that propagates the scientific knowledge in the scientific community. Before continuing, it is important to state what is meant with research or scientific paper. Robert Day ${ }^{1}$ defines a scientific paper as "a written and published report describing original research results", while acknowledging the fact that scientific papers also have to meet requirements regarding how the paper is written, and the way it is published. This means that the process leading to publication is equally important as the content, style, and organization of the published paper. Motives for writing and publishing a scientific paper can be outlined as (1) Scientific communication (2) Gain of reputation (3) Results worth reporting (4) Progression of scientific thought (5) Improve one's chance of promotion (6) Wider reach of one's results ${ }^{2,3}$.

Before writing a paper a few pertinent questions need to be answered:

1. Is the work correct for publication: Determining the correct work which needs publication is very important. It's not necessary that the work maybe new or unpublished. Even work that has been published innumerable times can be published again if there's a new perspective to it or if there is a good follow up results.

2. Target audience: It is very important to determine the target audience, whether it is the professionals or students, as the language of paper will vary accordingly.

3. Target Journal: It is very important to decide upon the journal for which the paper is intended, as each journal specializes in a specific area of research. Hence its readership varies. A proper choice of journal can make a larger impact of published paper.

\section{WRITING A PAPER:}

Before starting to write a paper it's important to organize one's thoughts and data. A systemic approach to write a paper maybe represented in the following flowchart ${ }^{4}$ : 


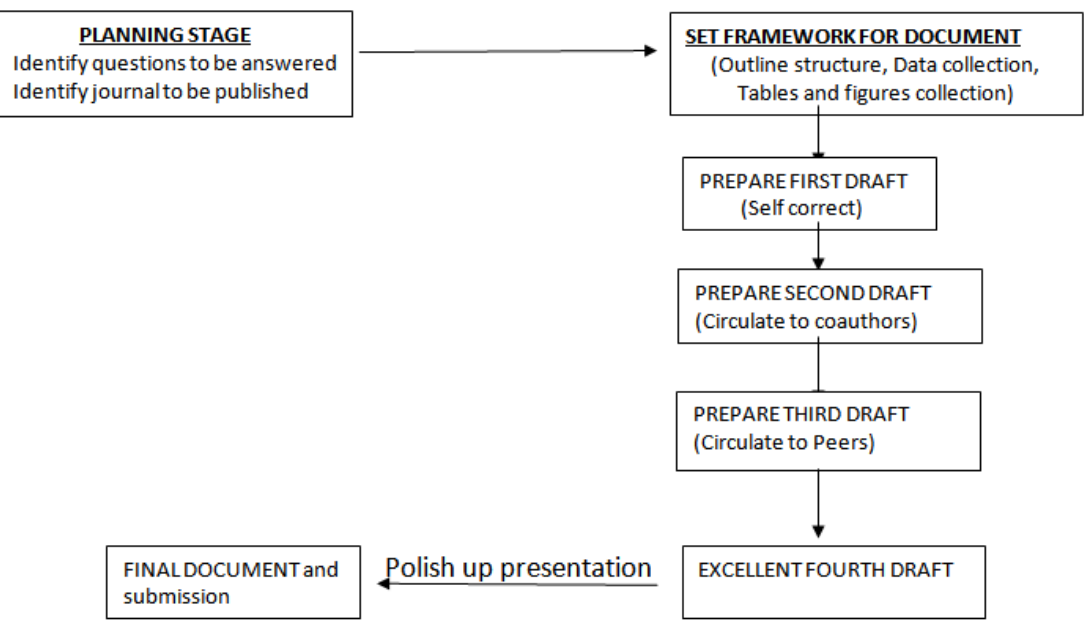

Plan for preparing and writing a paper for publication

\section{Structure of a Scientific Paper:}

The general structure of a paper comprises three major sections "hourglass model":

Introduction - The introduction leads the reader from general motivations and a general subject to a particular research question to be dealt with in the paper.

Body - The body of the paper stays within a tight thematic scope, as it describes the research methods, materials and results in detail.

Discussion- Discussion section tries to draw general conclusions from the particular results.

This smoothly aligns with Berry's claim ${ }^{5}$ that a research paper should be circular in argument, i.e., the conclusion should return to the opening, and examine the original purpose in the light of the research presented. However, there are additional parts of a paper with equal importance: Title, abstract, conclusion and the references.

Title

Abstract

Introduction

Body - Experimental Section, Materials and methods

Results and Discussion

Conclusions

References

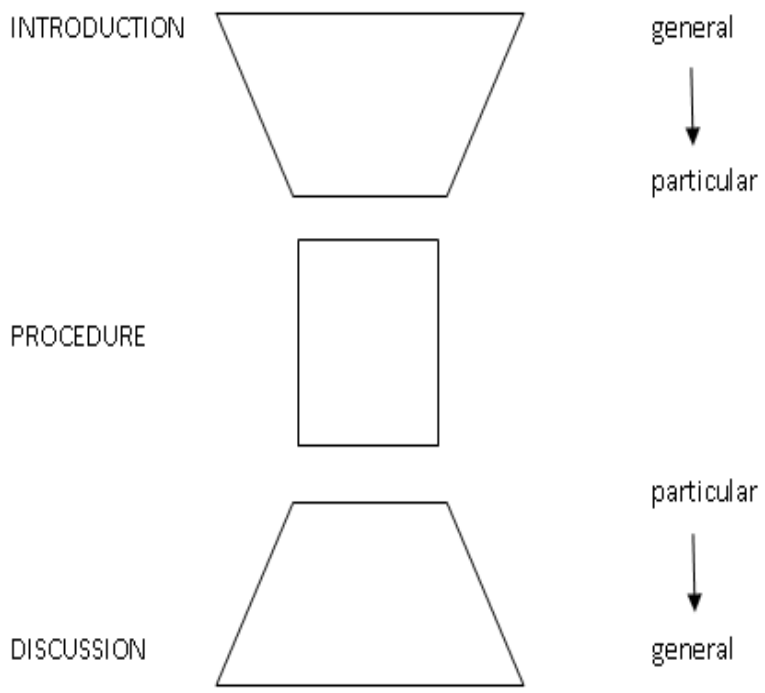

The hour-glass model of article structure (taken from Hill et al. 1982 (appearing in Swales 1990: 134))

TITLE:

Day ${ }^{1}$ defines a good title "as the fewest possible words that adequately describe the contents of the paper". 
Effective titles should ${ }^{3}$

1. Identify the main issue of the paper.

2. Begin with the subject of the paper.

3. Be accurate, unambiguous, specific, and complete,

4. Not contain abbreviations, and

5. Attract readers.

\section{ABSTRACT}

An abstract should be a very carefully prepared concise piece of prose simply describing the purpose of the study and the methods used, with a sentence outlining the results and where this fits into our knowledge. For the Journal of Bone and Joint Surgery the abstract need not be structured however, the Indian Journal of Orthopedics stresses on a structured abstract ${ }^{6}$. An abstract should focus on what the study is about. An ideal abstract should be ${ }^{7}$ :

1. Single paragraph and concise

2. As a summary of work done, it is always written in past tense.

3. An abstract should stand on its own, and not refer to any other part of the paper such as a figure or a table.

4. Focus on summarizing results - limit background information to a sentence or two, if absolutely necessary.

5. What the researcher report in an abstract must be consistent with what is reported in the paper.

6. Keywords - Using keywords is a vital part of abstract writing, because of the practice of retrieving information electronically: keywords act as the search term.

\section{INTRODUCTION:}

As already sketched above, the introduction section leads the reader from a general subject area to a particular field of research. The introduction is written in the present tense. Three phases of an introduction can be described ${ }^{8}$ :

1. Establish a territory- Define the problem:

a) Bring out the importance of the subject and/or

b) Make general statements about the subject and/or

c) Present an overview on current research on the subject.

2. Establish a niche - Describe what is already known about the problem:

a) Oppose an existing assumption or

b) Reveal a research gap or

c) Formulate a research question or problem or

d) Continue a tradition.

3. Occupy the niche - Describe your intent:

a) Sketch the intent of the own work and/or

b) Outline important characteristics of the own work;

c) Outline important results;

d) Give a brief outlook on the structure of the paper.

BODY:

The body of a paper reports on the actual research done to answer the research question or problem identified in the introduction. It describes what has already been done and is routinely the easiest part to write. The purpose of this section is basically to provide enough details so that a competent worker can repeat the experiments. This is the part that is most carefully reviewed. If your work involves other investigators who did part of the work, the paper should be written in collaboration with or after discussion with them. In a clinical study, start with why you selected the topic, what examinations were done, investigations carried out, and statistical methods employed. In experimental studies provide the technical specifications, the quantity, source and method of preparation. The chronology of events has to be followed strictly. It should be written as if it were an unfolding discussion, each idea at a time ${ }^{9}$.

Body of a paper consists of

a) Material and methods

b) Measurements 
c) Data analysis

Few important considerations while writing materials and methods:

1. How were the patients chosen, and what were the inclusion and exclusion criteria?

2. If the study required randomization, how was this achieved?

3. What tests were used?

4. What outcome scores were chosen and why?

5. It is important to identify who undertook the measurements or tests or outcome scores and whether they were blinded.

6. A clear description of the period of time during which the study was undertaken is required with an indication as to why this was chosen.

Statistics are a vital part of material and methods and helps in determining the significance of the study.

1. $P$-values indicate statistical significance.

2. Confidence intervals signifies clinical significance

\section{Discussion:}

The discussion and conclusion section is somehow the counterpart of the introduction, when thinking in terms of the hourglass model (cf. Figure 1), as this section leads from the particular results to general conclusions. Generally, this section includes ${ }^{1}$ :

1. Presentation of background information as well as recapitulation of the research aims of the present study.

2. Brief summary of the results, whereas the focus is on discussing not recapitulating the results.

3. Comparison of results with previously published studies.

4. Conclusions or hypotheses drawn from the results, with summary of evidence for each conclusion.

5. Proposed follow-up research questions.

Few things to remember while writing discussion ${ }^{10}$ :

1. Do not replicate the results section, but start with the major findings.

2. Point out exceptions or lack of correlation.

3. Show how the results and interpretations agree (or disagree) with previously published works. Then describe the theoretical and practical implications of the present study.

Common mistakes while writing discussion:

1. Failure to summarize

2. Unwarranted review of literature

3. Unwarranted conclusion not supported by data.

4. Extrapolation

\section{Result:}

The results section should be straight forward and clearly presented in a readily understandable fashion, with the appropriate use of tables and figures.

Things to remember while writing result:

1. Describe the results in detail and include a healthy, detailed discussion

2. The order of figures should follow the discussion themes and not the sequence they were conducted

3. Discuss how your data compare or contrast with previous results.

4. Include schemes, photographs to enhance the scope of discussion

5. Information in general should not be duplicated in the text and the tables.

In this section the numbers of patients, tests, outcome scores, length of follow-up etc, should match those described in the materials and method section.

\section{Conclusion:}

Include major findings followed by brief discussion on future perspectives and/or application of present work to other disciplines. It is important to not rewrite the abstract.

Refrences: 
Recognition and integrating one's work in scientific community is the principle aim of writing a research paper and this can easily be achieved by embedding ones work in the related literature. So there will be citations of references in the text, as well as a list of cited references at the end of the paper.

\section{CITING A SOURCE}

1. Paraphrasing - Rephrasing an authority's words. Ideas taken from other sources and put in one's own words must be cited to give credit to the sources.

2. Quoting is rewriting, word-for-word, someone else's words. One must pay close attention to the original wording, spelling, and punctuation, and follow them exactly.

Different systems are used for referencing. The most commonly used referencing systems are:

a) In text citation (Name and Year System): References are cited by their respective authors and the year of publication, e.g., "Leo et al (2012) says...."

This system is very convenient for authors, as the citation does not have to be changed when adding or removing references from the list. Though with this method; sentences become hard to read when many references are cited in one single parenthesis.

b) Reference List (Bibliography)

- Numerically (Vancouver style): This system lists the references in the order of appearance (citation by number) in the text and cites them by their respective number

Bibliographic Elements in parentheses or (square) brackets, e.g., "As reported in (4),..."

- Authors (use et al. after 6 authors, if there are more than six authors, complete names should not be written. "et al" must be in italics)

- Article title (should be exact as existing)

- Journal name (should be in standard abbreviations, full journal name should not be written)

- Year

- Volume

- Page numbers

- Alphabetically (Harvard style): This system lists the references according to the author's last name if using an author-date style.

This system is relatively convenient as it does not break the flow of words while reading a sentence with many citations.

\section{TYPES OF REFRENCES:}

a. Journal Reference

b. Book Reference

c. Internet Reference

\section{A) JOURNAL REFRENCE:}

Two main components of a Journal Reference

i. Bibliographic Elements

ii. Punctuations Marks

\section{BIBLIOGRAPHIC ELEMENTS}


Authors in correct sequence

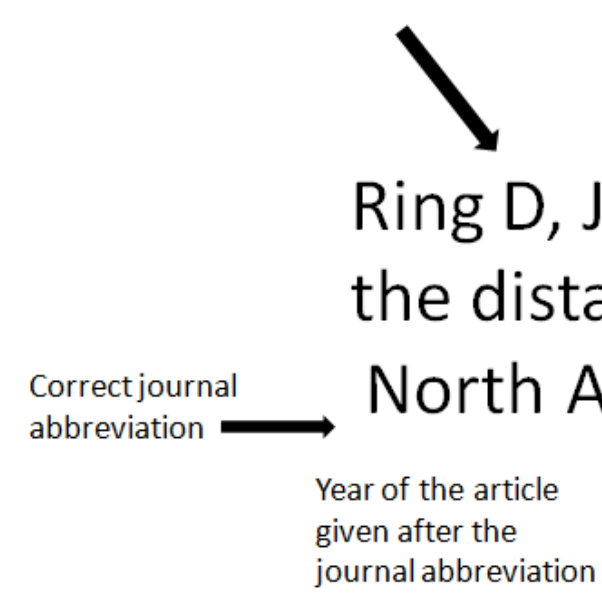

Correct journal abbreviation

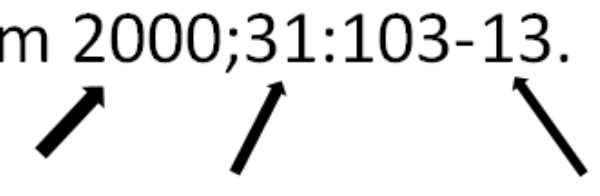

Correct title of the article to come after the authors name.

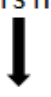

Fractures of Orthop Clin

Year of publication is followed by the volume no.

PUNCTUATION MARKS

\section{Punctuation Marks}

Coma followed by space after each author do not write and before the last author
Full stop after the last author followed by space and then the article title

Ring D, Jupiter JB.

Fractures of the distal humerus. Full stop followed by space

Orthop Clin Full stop at the North Am 2000;31:103-13. end of the ref.

Single space between the journal abbreviation and the year, place no other punctuation marks

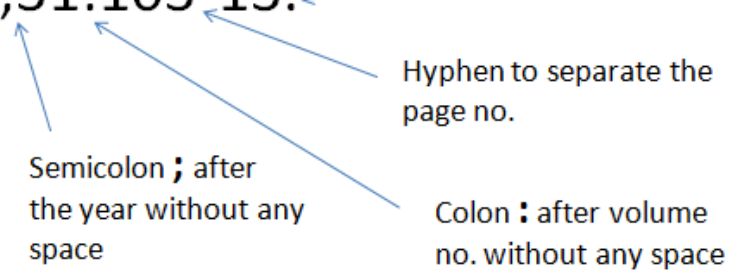

B) BOOK REFRENCE: Chapter in a book. 


\section{City of publication}

\section{Mivano T, Kobayashi $\mathrm{H}$, Chen SC. Long term} results of biliary atresia. In: Gupta DK, editor.

$\left.\begin{array}{l}\text { Text Book } \\ \text { Delhi: Moc of the } \\ \text { book }\end{array}\right\}$

$\left.\begin{array}{l}\text { Text Book } \\ \text { Delhi: Moc of the } \\ \text { book }\end{array}\right\}$

\section{Name of} publisher

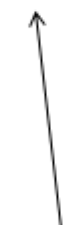

\section{urgery, $1^{\text {st }}$ ed. New} rs; 2000 . p. 288-91.

C) INTERNET REFRENCE:

The three main types of electronic sources are:

1. Websites or web pages :

Homepage/Web site

- Cancer-Pain.org [Internet]. New York: Association of Cancer Online Resources, Inc.; c2000-01 [updated 2002 May 16; cited 2002 Jul 9]. Available from: http://www.cancer-pain.org/.

2. Complete works (reports, research papers, online books)

Database on the Internet

- Who's Certified [Internet]. Evanston (IL): The American Board of Medical Specialists. c2000 - [cited 2001 Mar 8]. Available from: http://www.abms.org/newsearch.asp

3. Journal article on the Internet

- Abood S. Quality improvement initiative in nursing homes: the ANA acts in an advisory role. Am J Nurs [Internet]. 2002 Jun [cited 2002 Aug 12];102(6):[about 1 p.]. Available from: http://www.nursingworld.org/AJN/2002/june/Wawatch.htmArticle

Few things to remember while citing references ${ }^{10}$ :

1. List significant published references.

2. Unpublished references should be cited only if necessary.

3. All the references should be complete, with author name(s), year, article title, journal name, page extent, and publisher, and should match the text citation.

4. Avoid cross referencing from other articles; check it yourself, if available in your library.

5. Choose references that strengthen your argument

While writing a scientific paper one should have basic understanding of certain facts regarding publications:

a) Authorship 
b) Plagiarism

c) Impact factor

Authorship: An "author" is generally considered to be someone who has made substantive intellectual contribution to a published study. The name that appears at the beginning of a paper serves an important purpose. It let others know who conducted the study and should get credit for it. Likewise, the credit derived from publications is used to determine an author's worth.

Academic Performance Indicator (API) for publications is carried out in following manner as per the UGC (University Grants Commission, New Delhi)

1. Single author $-100 \%$ of total points

2. Two Authors(First/Principal author + the corresponding author/supervisor/mentor) $-60 \%$ of the total points to each.

3. Three or more authors (First/Principal author + the corresponding author/supervisor/mentor + others)$60 \%$ of the total points to each First/Principal author + the corresponding author/supervisor/mentor \& $40 \%$ of the total points to all others

Plagiarism: One should not claim words and ideas of another as their own; rather should give credit where credit is due. Whether paraphrasing, quoting an author directly, or describing an idea that influenced one's work, the source should always be credited. Quoting somebody without giving proper credit can lead to plagiarism.Plagiarism is not a crimeper se but in academia; it is a serious ethical offense.

Impact Factor: The measure of quality and prestige of a journal is the impact factor ${ }^{11}$. It is used for ranking and evaluating the journals and also to judge the academic performance and the quality and importance of an individual research publication. The IF is a product of the Institute of Scientific Information (ISI) of Philadelphia, PA. ISI founded in 1958, is an independent, non-governmental private company. This has a database which lists the contents of scientific journals published worldwide. They calculate the number of citation of each paper within the database receives from other papers within the database. A paper which receives more citation is valued more.

The IF for a journal is calculated by dividing the number of citation received in a particular year for the number of articles published in preceding 2 years. This gives a numeric value. To calculate the IF of Indian Journal of Orthopaedicsin the year 2011, the citations received in the year 2011 forthe articles published in year 2009 and 2010 (numerator)will be divided by number of articles published in year 2009and 2010 (denominator).

IF in 2011= Citation in 2011 for the articles published in 2009 and 2010

Number of articles published in 2009 and 2010

This IF of a journal is intended to measure how often, on an average, authors cite moderately recent articles from the particular journal.4 The original papers, notes and review articles are included in the total of denominator while citation of original articles, notes, review articles, editorial, letters and meeting abstracts are included as numerator. The IF appearing in 2012 will reflect IF of 2011 of any journal for articles published in 2009 and 2010.The IF for Indian journal of Orthopedics is listed in journal citation report (JCR) published around July-August every year.

Some people have a flair for writing, some with all the experience and knowledge in their minds just can't reproduce same on the paper. Even experienced scholars are noted to be not very good in paper writing.

\section{THINGS TO BE AVOIDED:}

Ten characteristics of an incredibly dull paper Sand-Jenson in Oikos 2007, 116723 (C\&E News Sept 10, 2007)

1. Loss of focus; unnecessary details.

2. No originality and personality.

3. Really long article.

4. No indication of any potential implications.

5. No illustrations.

6. Left out necessary steps of reasoning.

7. Use of abbreviations and technical terms that only specialists in the field can understand.

8. Too serious paper with no significant discussion. 
9. Sole focus only on statistics.

10. Every statement supported with a reference.

\section{Bibliography:}

[1] Day RA. How to Write and Publish a Scientific Paper.1983.Second edn. ISI Press, Philadelphia

[2] O'Connor M. Writing Successfully in Science.1995.Chapman \& Hall, London

[3] Peat J, Elliott E, Baur L, Keena V. Scientifc Writing - Easy when you know how. BMJ Books, London (2002)

[4] Davis M. Scientific Papers and Presentations.1997.Academic Press, SanDiego

[5] Berry R. How to Write a Research Paper. Second edn. Pergamon Press, Oxford(1986)

[6] Scott James. How to write a paper for a journal. IJO 2007 ;41:55-56

[7] Bhakar, S.S., Mehta Seema: A Systematic Guide to Write a Research Paper

[8] Swales, J.M.: Genre analysis: English in academic and research settings.1993. Cambridge Univ. Press, Cambridge

[9] Dees R. Writing the Modern Research Paper. 1997.Secondedn. Allyn\& Bacon, Boston

[10] Biswas J. Practical suggestions in the writing of a research paper. Indian J Ophthalmol 1998; 46: 247-50

[11] Anil K Jain. Impact factor: Measure of quality of research publication; Indian J Orthop. 2011 Jul-Aug; 45(4): 289-290. 